Article

\title{
Consequences of $f(\mathcal{T})$ Cosmology in Thermal Leptogenesis and Gravitino Late Abundance
}

\author{
Antonio Capolupo ${ }^{1}$, Salvatore Marco Giampaolo ${ }^{2}$, Gaetano Lambiase ${ }^{1, *}$ and \\ Aniello Quaranta ${ }^{1}$ \\ 1 Dipartimento di Fisica “E.R. Caianiello" Universitá di Salerno, and INFN-Gruppo Collegato di Salerno, \\ SA 84084 Fisciano, Italy; capolupo@sa.infn.it (A.C.); aquaranta@unisa.it (A.Q.) \\ 2 Division of Theoretical Physics, Ruder Bošković Institute, Bijenčka cesta 54, 10000 Zagreb, Croatia; \\ sgiampa@irb.hr \\ * Correspondence: glambiase@unisa.it
}

Received: 31 January 2020; Accepted: 11 February 2020; Published: 19 February 2020

\begin{abstract}
Thermal Leptogenesis and the gravitino problem are reviewed in the framework of non-standard cosmologies. We consider in particular the $f(\mathcal{T})$ cosmology, where $\mathcal{T}$ is the torsion field. We constrain the parameters space of these cosmological models consistently with thermal Leptogenesis scenario (with degenerate mass spectrum of light neutrinos), and we show that they allow to solve the gravitino problem as well. Owing to the similar characteristics to $f(\mathcal{T})$ cosmology, we shortly discuss also the case of the shear dominated Universe.
\end{abstract}

Keywords: Early Universe; Modified Cosmology; Leptogenesis

\section{Introduction}

Modified theories of gravity have been proposed in the last years as a possible solution of the shortcomings related to the Cosmological Standard Model, based on General Relativity (GR). This is the case, for example, of higher order curvature invariants than the simple Ricci scalar $R$ that allow to get inflationary behaviors, removing the primordial singularity, and to explain the flatness and horizon problems [1] (see also [2-18]). This approach and, of course, all those related to it, are fundamentally motivated by the fact that, at high curvature regimes, further curvature invariants have to be considered for constructing self-consistent effective actions in curved spacetime [19-21]. In this respect, modified gravity could provide the unification of the early-time (inflation) and the later-time acceleration of the Universe [22,23], providing at the same time, an unified description of Dark Energy (DE) and Dark Matter (DM).

One of the consequences to deal with the non standard cosmology is that the thermal history of particles gets modified. In fact, the expansion rate of the Universe can be typically written in the form [24],

$$
H(T)=A(T) H_{G R}(T),
$$

where $H_{G R}$ is the expansion rate of the Universe in GR, and $A(T)$ accounts for deviations from GR. The latter is defined such that the successful predictions of the abundance of primordial light elements is preserved, i.e., $A(T) \neq 1$ at early time, or equivalently, at temperatures $T>T_{B B N}$, where $T_{B B N}$ is the big bang nucleosynthesis (BBN) temperature, and $A(T) \rightarrow 1$ before or when the BBN begin. Therefore, it is common to refer to the so-called pre-BBN epoch, since this era is not directly constrained by cosmological observations. Typically this factor is parameterized as [24]

$$
A(T)=\eta_{*}\left(\frac{T}{T_{*}}\right)^{v}
$$


where $T_{*}$ is a parameter with dimensions of the temperature (interpreted as the transition temperature from the modified cosmology to the standard cosmology), and $\left\{\eta_{*}, v\right\}$ are free parameters. All these parameters do depend on the cosmological model under consideration. Investigations along these lines have been performed in different cosmological scenarios [24,25]: $(i) . v=2$ in Randall-Sundrum type II brane cosmology [26]; (ii). $v=1$ in kination models [27]; (iii). $v=0$ in cosmologies with an overall boost of the Hubble expansion rate [24,28-34]; (iv). Gauss-Bonnet scenario $v=-2 / 3$ [35]; $(v) . v=-0.8$ in scalar-tensor cosmology [24,36]; (vi). $v=2 / n-2$ in $f(x)$ cosmology, with $f(x)=$ $x+\alpha x^{n}$ and $x=R, \mathcal{T}$, where $R[2,4]$ and $\mathcal{T}$ [37-39] stand for the scalar curvature and the scalar torsion, respectively.

The aim of this paper is twice. First we study the origin of the matter-antimatter asymmetry in the Universe in the case in which the evolution of the Universe is described by non-standard cosmologies. The matter-antimatter asymmetry represents one of the open issue of the modern cosmology and particle physics. The successful predictions of BBN combined with the observations of the $\mathrm{CMB}$ anisotropies show that the parameter that characterizes such a asymmetry is $\eta=$ $\frac{n_{B}-n_{\bar{B}}}{s}<(9.2 \pm 0.5) \times 10^{-11}$, where $n_{B}\left(n_{\bar{B}}\right)$ is the baryon (antibaryon) number and $s=\frac{2 \pi^{2} g_{*} T^{3}}{45}$ the entropy density ( $g_{*}=106.7$ counts the total degrees of freedom for the relativistic particles). Among the models proposed in literature for explaining the origin of baryon/antibaryon asymmetry, the thermal Leptogenesis is certainly one of the favorite candidates. The asymmetry is generated in the lepton sector, and through non-perturbative electroweak processes involving sphalerons physics, is totally or partially converted to an asymmetry in the baryon sector. Leptogenesis is the simplest extension of the Standard Model (SM) of particle physics that includes right handed neutrinos $N_{R}$. They implement the see-saw mechanism, providing from a side a possible explanation of light neutrinos mass of the SM, from the other side to generate the lepton asymmetry from the decays of right handed neutrinos. Moreover, Leptogenesis is very attractive since allows a possible lepton number violation in the neutrino sector [40,41] (for a recent review see Ref. [42-44]), as well as the Sakharov conditions for explaining the matter-antimatter asymmetry are easily realized.

In thermal Leptogenesis, the out-of-equilibrium condition occurs when $\Gamma_{N_{1}}<H_{G R}$ at $T=$ $M_{1}$, where $\Gamma_{N_{i}}=\frac{\left(\lambda^{+} \lambda\right) M_{1}}{8 \pi}$ is the decay rate of heavy Majorana right-handed neutrinos, $H_{G R}=$ $\sqrt{\frac{8 \pi^{3} g_{*}}{45}} \frac{T^{2}}{M_{P l}}, M_{1}$ is the heavy right-handed neutrino mass, and finally $\lambda_{i k}$ are the complex Yukawa coupling constants (such a study for the brane cosmology has been performed in [45]). In terms of the scaled quantities

$$
\begin{aligned}
\tilde{m}_{1} & \equiv \frac{8 \pi v^{2}}{M_{1}^{2}} \Gamma_{N_{1}}=\frac{\left(\lambda^{\dagger} \lambda\right) 11 v^{2}}{M_{1}} \\
m_{*} & \equiv \frac{8 \pi v^{2}}{M_{1}^{2}} H_{G R}\left(T=M_{1}\right) \simeq 1.1 \times 10^{-3} \mathrm{eV}
\end{aligned}
$$

where $\left(\lambda^{\dagger} \lambda\right)_{11}=\sum_{j}\left(\lambda_{1 j}^{\dagger} \lambda_{1 j}\right)$, the out-of equilibrium condition reads

$$
\tilde{m}_{1}<m_{*} \simeq 10^{-3} \mathrm{eV} .
$$

This condition provides an upper bound on the lightest neutrino mass $m_{1}$ owing to the inequality $m_{1} \leqslant \tilde{m}_{1}$ [46-49]. Such a bound normally implies that thermal Leptogenesis cannot generate sufficient baryon asymmetry for degenerate masses of light neutrinos, as immediately follows from the expression of the $\mathrm{CP}$ parameter $\epsilon \simeq 10^{-6} \sin \delta \frac{m_{3}}{5 \times 10^{-2} \mathrm{eV}} \frac{M_{1}}{10^{10}} \mathrm{GeV}$ entering the lepton asymmetry $\eta \sim \epsilon$, see Appendix A. As we will show, in the case in which the cosmological background evolves according to (1), hence for modified cosmologies, the bound (5) turns out to be $\tilde{m}_{1}<m_{*} A(T)$. As a 
consequence, the parameters characterizing non-standard cosmologies can be constrained by thermal Leptogenesis in the case of degenerate light neutrino mass spectrum.

Moreover, we also explore the implications of modified cosmologies in relation to the gravitino problem (see for example [50]). Gravitino couples to ordinary matter only through the gravitational interaction, with a (quite) long lifetime $\tau_{3 / 2} \sim \frac{M_{P l}^{2}}{m_{3 / 2}^{3}} \simeq 10^{5}\left(\frac{1 \mathrm{TeV}}{m_{3 / 2}}\right)^{3}$ sec, where $m_{3 / 2}$ is the mass of the particle $\sim 10^{2} \mathrm{GeV}$. The decay of these particles with such a long lifetime is problematic in cosmology since their decay products would destroy light elements, destroying the successful predictions of BBN. This problem can be avoided by putting an upper bound on the reheating temperature, that for the standard cosmological model is given by $T_{R} \lesssim\left(10^{6}-10^{7}\right) \mathrm{GeV}$ for $m_{3 / 2} \sim \mathcal{O}\left(10^{2} \mathrm{GeV}\right)$. It is worth to mention another mechanism to generate gravitinos by means of primordial black holes (PBHs) evaporation [51]. As shown in [51], as source of gravitions is also provided by the evaporation of light PBHs, with initial $M_{i} \lesssim 10^{9}$ gr. The subsequent decay of evaporated gravitinos into cascades of non-equilibrium particles, as occurs in minimal supergravity, leads to the formation of elements whose abundance is constrained by observations, and one has to require that their density does not overclose the Universe. A consequence of this mechanism is that cosmological models with substantial inhomogeneities on small scales are excluded.

This bound opens a serious question for the inflationary models (and for some models of Leptogenesis) since these models predict a larger reheating temperature [50]. Such a difficulty can be avoided, as we will see, in the framework of non-standard cosmologies.

The paper is organized as follows. In next Section we discuss the thermal Leptogenesis for degenerate mass spectrum of the light neutrinos in the context of non-standard cosmologies. The gravitino problem is also discussed. Here we provide a general discussion considering non-standatd cosmologies characterized by a Friedamn equation of the form $H^{2} \sim \rho^{n}$. In Section 3 we analyzed specific examples of non-standard cosmologies, focusing in particular on Teleparallel Equivalent of General Relativity and shear dominated Universe. Conclusions are discussed in Section 4.

\section{Thermal Leptogenesis in Modified Cosmologies}

We consider the case in which the lightest heavy neutrino $N_{1}$ decays during the phase of the Universe expansion described by the modified cosmology. The expansion rate of the Universe is therefore given by (1) with $A(T)$ defined in (2). In such a case, the out-of-equilibrium condition reads

$$
\Gamma\left(T=M_{1}\right)<H\left(T=M_{1}\right)=H_{G R}\left(M_{1}\right) A\left(M_{1}\right),
$$

or equivalently, in terms of the scaled masses, Equation (5) assumes the form

$$
\tilde{m}_{1}<m_{*} A(T) \simeq 10^{-3} A(T) \mathrm{eV} .
$$

The upper bound turns out to be hence modulated by the factor $A(T)$. It then follows that for low temperatures, i.e., $T_{*}<T \lesssim M_{1}$, the non-standard cosmologies are consistent with thermal Leptogenesis with a degenerate light neutrino mass spectrum. In fact one gets

$$
K_{1} \equiv \frac{\Gamma_{N_{1}}}{H\left(T=M_{1}\right)}=\frac{1}{A\left(M_{1}\right)} \frac{\Gamma_{N_{1}}}{H_{G R}\left(T=M_{1}\right)}=\frac{1}{A\left(M_{1}\right)} \frac{\tilde{m}_{1}}{m_{*}}=\frac{1}{A\left(M_{1}\right)} \frac{\tilde{m}_{1}}{10^{-3} \mathrm{eV}}<1 \quad \text { for } \quad A\left(M_{1}\right) \gg 1,
$$

so that, the effects of modified gravity is to avoid the suppression of Leptogenesis even in the case of degenerate light neutrino masses, determining a sufficient baryon asymmetry.

The above discussion relies on the assumption that the neutrinos $N_{1}$ are in thermal equilibrium at temperature $T$ greater than the heavy Majorana neutrino mass $M_{1}, T>M_{1}$ (this assumption underlies the derivation of (7)). We shall now verify that such an assumption can be realized in modified cosmologies. Owing to the fact that $N_{1}$ are singlets under the Standard Model gauge group, the only 
interaction that allows to $N_{1}$ to be in thermal equilibrium is the Yukawa coupling (see Equation (A1) in Appendix A). The thermal average (pair) annihilation rate is [45]

$$
\Gamma_{a n n}(T)=n_{N_{1}}\langle\sigma v\rangle \simeq \frac{3 \xi(3) T^{3}}{2 \pi^{2}} \frac{N_{a n n} \lambda^{4}}{a_{K i n} T^{2}}
$$

where $N_{a n n}$ is the number of annihilation channels, $\lambda$ denotes the dominant Yukawa's coupling, $a_{\text {Kin }} \sim 10^{2}$ is the kinematical phase factor, and $N_{a n n} \lambda^{4} \simeq \mathcal{O}(10)$. The freeze-out temperature $T_{f}$, determined as usual by $\Gamma_{a n n}\left(T_{f}\right)=H\left(T_{f}\right)=H_{G R}\left(T_{f}\right) A\left(T_{f}\right)$, is given by

$$
\begin{aligned}
T_{f} & =\left[\frac{3 \xi(3)}{2 \pi^{2} \eta_{*}} \frac{N_{a n n} \lambda^{4}}{a_{K i n}} \sqrt{\frac{45}{8 \pi^{3} g_{*}}}\right]^{\frac{1}{v+1}}\left(\frac{T_{*}}{M_{P l}}\right)^{\frac{v}{v+1}} M_{P l} \\
& \simeq\left(\frac{7.5 \times 10^{-3}}{\eta_{*}} \frac{M_{P l}}{T_{*}}\right)^{\frac{1}{v+1}} \frac{T_{*}}{\mathrm{GeV}} \mathrm{GeV}
\end{aligned}
$$

In order that thermal Leptogenesis can be realized in modified cosmologies, one has to require that the mass of Majorana neutrinos is such that

$$
T_{*}<M_{1} \lesssim T_{f} .
$$

In what follows we shall assume $M_{1} \simeq\left(10^{8}-10^{9}\right) \mathrm{GeV}$ (see Appendix A).

\subsection{Kinetic Equations}

Here we shortly discuss the kinetics equations in the framework of non standard cosmology. The kinetic equation for the leptogenesis are given by (see for example [52])

$$
\frac{d N_{1}}{d z}=(D+S)\left(N_{1}-N_{1}^{e q}\right), \quad \frac{d N_{B-L}}{d z}=-\epsilon D\left(N_{1}-N_{1}^{e q}\right)-W N_{B-L},
$$

with $z=M_{1} / T,(D, S, W)=\left(\Gamma_{D}, \Gamma_{S}, \Gamma_{W}\right) /(H z)$, where $\Gamma_{D, S, W}$ are the scattering rates defined as $\Gamma_{D}=\frac{M_{1}}{8 \pi}\left(\lambda^{\dagger} \lambda\right)_{11} \frac{K_{1}(z)}{K_{2}(z)}\left(K_{1,2}(z)\right.$ are the Bessel functions), $\Gamma_{S}=2 \Gamma_{N_{1} \rightarrow \phi, t}+4 \Gamma_{N_{1} \rightarrow \phi, s}\left(\Gamma_{N_{1} \rightarrow \phi,\{t, s\}}\right.$ are the $N_{1}$ scattering rates in which the Higgs $\phi$ processes in $t$ - and s-channels are involved), and $\Gamma_{W}$ is the washout-rate. The quantities $\Omega=D, S, W$ are proportional to $\lambda^{\dagger} \lambda$, so that the reduced rates entering (12) are such that [52] $\Omega-\Delta W \propto \frac{M_{P l} \tilde{m}_{1}^{D}}{v^{2}}$, where $\Delta W \propto \frac{M_{P l} \bar{m}^{2} M_{1}}{v^{4}}$, with $\bar{m}^{2} \simeq 3 m_{i}^{2}$ for quasi degenerate neutrinos, and $\tilde{m}_{1}^{D}=\frac{\left(m_{D}^{+} m_{D}\right) 11}{M_{1}}$, with $m_{D}$ the Dirac neutrino mass. The latter equation shows that the dependence of the generated lepton asymmetry on $M_{1}$ is relaxed as long as $\Delta W$ is negligible. The Boltzamann equations of the number of heavy Majorana neutrinos $N_{1}$ and of $B-L$ number $N_{B-L}$, Equation (12), are dependent on $A(T)$. However, in this paper we are mainly interested to fulfill the conditions allowing the out-of-equilibrium, and the consequent constraints on the parameter spaces of the non-standard cosmology, therefore the numerical analysis of the $N_{1}$ and $N_{B-L}$ quantities are beyond the scope of the present paper, and will be faced elsewhere (in the regime to which we are interested in this paper, i.e., $A(T) \sim T(v=1)$ and $A\left(T \simeq M_{1}\right) \sim \mathcal{O}\left(10-10^{2}\right)$, see next Section, one gets $\frac{H(z)}{H(z=1)}=\frac{1}{z} \frac{H_{G R}(z)}{H_{G R}(z=1)}, \frac{\Gamma_{D, S, W}}{H(z)}=\frac{z}{A\left(M_{1}\right)} \frac{\Gamma_{D, S, W}}{H_{G R}(z)}$, while the abundance of $N_{1}$ and $N_{B-L}$ in non-standard cosmologies may remain unaffected, with respect results obtained in the standard cosmological model [52], for appropriate initial conditions). 


\subsection{The Gravitino Problem}

The gravitino abundance is described, in the standard cosmological model, by the Boltzmann equation (here we follow Ref. [45], where the gravitino problem has been discussed in the framework of Brane Cosmology)

$$
\frac{d Y_{3 / 2}}{d T}=-\Theta_{G R}, \quad \Theta_{G R} \equiv \frac{s\langle\sigma v\rangle Y_{\text {rad }}^{2}}{H_{G R} T},
$$

where $Y_{3 / 2}=n_{3 / 2} / s$ the gravitino abundance, $Y_{\text {rad }}=n_{\text {rad }} / s$ the radiation abundance, and $s$ the entropy density, it follows $Y_{3 / 2} \simeq 10^{-11} \frac{T_{R}}{10^{10} \mathrm{GeV}}$, where $T_{R}$ is the reheating temperature. The adiabatic evolution of the Universe and the conservation of the entropy is assumed. Note that the quantity $\Theta_{G R}$ is independent on $T$ since $\langle\sigma v\rangle \sim M_{P l}^{-2}, H_{G R} \sim T^{2} / M_{P l}$, and $s \sim T^{3}$. To solve the gravitino problem one has to impose that the reheating temperature is constrained to $T_{R} \lesssim\left(10^{6}-10^{7}\right) \mathrm{GeV}$ (for gravitino masses of the order of $100 \mathrm{GeV}$ ) [50]. This bound is problematic for thermal Leptogenesis in supersymmetric models after inflation since in these frameworks the reheating temperature is many order of magnitudes greater than $\left(10^{6}-10^{7}\right) \mathrm{GeV}[50,53-55]$.

However, if the Universe evolution is described by a non standard cosmology, then the expansion rate is replaced by (1). This implies that the factor $\Theta_{G R}$ in (13) is replaced by $\Theta_{G R} \rightarrow \Theta=\Theta_{G R} / A(T)[45,56,57]$. In such a circumstance, the reheating temperature $T_{R}$ entering the above expression for $Y_{3 / 2}$ is replaced by the transition temperature $T_{*}$. In fact, referring to cosmological models studied in the next Sections, $v=1$ and $v>1$, the integration of (13) from $T_{*}$ to $T_{R}$ (assuming $T_{R} \gg T_{*}$ and using (2)), gives

$$
Y_{3 / 2} \sim \begin{cases}\frac{T_{*}}{\eta} \log \frac{T_{R}}{T_{*}} & v=1 \\ \frac{T_{*}^{2 v-1}}{\eta(v-1)} & v>1\end{cases}
$$

Therefore, in the case in which the transition temperature is low enough, i.e., $T_{*}<\left(10^{6}-10^{7}\right) \mathrm{GeV}$, the gravitino problem is avoided even if the reheating temperature is much higher.

Let us check that the condition $T_{R} \gg T_{*}$ occurs also in non-standard cosmology. By comparing the expansion rate of the Universe (1) with the decay rate of the inflaton $\Gamma_{I}$ one obtains the reheating temperature

$$
T_{R}=T_{*}\left(\frac{M_{P l} \Gamma_{I}}{T_{*}^{2}}\right)^{\frac{1}{v+2}} .
$$

On the other hand, in the standard cosmology, the relation $H_{G R}\left(T_{R}^{G R}\right)=\Gamma_{I}$ gives $T_{R}^{G R} \simeq\left(M_{P l} \Gamma_{I}\right)^{1 / 2}$. Combining this relation with the reheating temperature (14), one infers

$$
T_{R}=T_{*}\left(\frac{T_{R}^{G R}}{T_{*}}\right)^{\frac{2}{v+2}} .
$$

Thus $T_{R} \gg T_{*}$ provided $T_{R}^{G R} \gg T_{*}$, as in the standard cosmology (such a relation, however, is such that the values $v \gg 1$ are excluded).

\section{Examples of Non Standard Cosmologies}

As pointed out, results of previous Section are general, and apply to cosmological models in which the expansion rate can be cast in the form of Equations (1) and (2). In this Section we shall consider some specific models of modified cosmologies. We first focus on $f(\mathcal{T})$ model, deriving a bound on the parameter of the model, then we shortly discuss the shear dominated Universe $(v=1)$. 


\section{1. $f(\mathcal{T})$ Cosmology}

An interesting cosmological model recently proposed in literature is the Teleparallel Equivalent of General Relativity (TEGR) [37]. This theory of gravity is based on the Weitzenböck connection (instead of the usual Levi-Civita connection), and the gravitational field is described by the torsion tensor (instead of the curvature tensor)

$$
\mathcal{T}_{\mu \nu}^{\lambda}=\hat{\Gamma}_{\nu \mu}^{\lambda}-\hat{\Gamma}_{\mu \nu}^{\lambda}=e_{i}^{\lambda}\left(\partial_{\mu} e_{v}^{i}-\partial_{\nu} e_{\mu}^{i}\right)
$$

where $e_{\mu}^{i}(x)$ are the vierbein fields defined as $g_{\mu v}(x)=\eta_{i j} e_{\mu}^{i}(x) e_{\nu}^{j}(x)$. The action is given by

$$
S_{I}=\frac{1}{16 \pi G} \int d^{4} x e[\mathcal{T}+f(\mathcal{T})]
$$

where $\mathcal{T}=S_{\rho}^{\mu \nu} \mathcal{T}_{\mu \nu}^{\rho}$ is the torsion scalar, $e=\operatorname{det}\left(e_{\mu}^{i}\right)=\sqrt{-g}, f(\mathcal{T})$ is a generic function of the torsion, and

$$
S_{\rho}^{\mu v}=\frac{1}{2}\left[\frac{1}{4}\left(\mathcal{T}^{\mu v}{ }_{\rho}-\mathcal{T}^{v \mu}{ }_{\rho}-\mathcal{T}_{\rho}^{\mu v}\right)+\delta_{\rho}^{\mu} \mathcal{T}^{\theta v}{ }_{\theta}-\delta_{\rho}^{v} \mathcal{T}^{\theta \mu}{ }_{\theta}\right] .
$$

TEGR represents an alternative to inflation models, as well as to effective DE models, in which the Universe acceleration is driven by the torsion terms $[37,58]$ (see the review $[59,60]$ ). For a homogeneous and isotropic Universe (FRW Universe) one finds that $e_{\mu}^{A}=\operatorname{diag}(1, a, a, a)$ and $\mathcal{T}=-6 H^{2}$. The cosmological field equations are [60]

$$
\begin{gathered}
12 H^{2}\left[1+f_{\mathcal{T}}\right]+[\mathcal{T}+f]=16 \pi G \rho, \\
48 H^{2} f_{\mathcal{T} \mathcal{T}} \dot{H}-\left(1+f_{\mathcal{T}}\right)\left[12 H^{2}+4 \dot{H}\right]-(\mathcal{T}-f)=16 \pi G p,
\end{gathered}
$$

where $f_{\mathcal{T}}=d f / d \mathcal{T}$. As an explicit example, we consider the power-law $f(T)$ model $f(\mathcal{T})=$ $\beta_{\mathcal{T}}|\mathcal{T}|^{n_{\mathcal{T}}}$ [61,62]. By rewriting (19) in the form $H_{G R}^{2}+H_{\mathcal{T}}^{2}=\frac{8 \pi}{3 M_{P l}^{2}} \rho$, where $H_{\mathcal{T}}^{2} \equiv \frac{f}{6}-\frac{T f_{\mathcal{T}}}{3}=$ $6^{n-1} \beta_{\mathcal{T}}\left(2 n_{\mathcal{T}}+1\right) H^{2 n}$, and assuming $H_{\mathcal{T}} \gg H_{G R}$, one gets $H_{\mathcal{T}}=A(T) H_{G R}$, where $A(T)$ is of the form (2) with

$$
\begin{aligned}
\eta & =1, \quad v=\frac{2}{n_{\mathcal{T}}}-2 \\
T_{*} & \equiv\left(\frac{24 \pi^{3} g_{*}}{45}\right)^{\frac{1}{4}}\left(2 n_{\mathcal{T}}+1\right)^{\frac{1}{4\left(1-n_{\mathcal{T}}\right)}}\left(\frac{\beta_{\mathcal{T}}}{\mathrm{GeV}^{2\left(1-n_{\mathcal{T}}\right)}}\right)^{\frac{1}{4\left(1-n_{\mathcal{T}}\right)}}\left(\frac{M_{P l}}{\mathrm{GeV}}\right)^{\frac{1}{2}} \mathrm{GeV} .
\end{aligned}
$$

From (20) it follows

$$
A\left(T=M_{1}\right)=\left(\frac{1}{\left(2 n_{\mathcal{T}}+1\right)} \frac{\mathrm{GeV}^{2\left(1-n_{\mathcal{T}}\right)}}{\beta_{\mathcal{T}}}\right)^{\frac{1}{2 n_{\mathcal{T}}}}\left[\left(\frac{45}{24 \pi^{3} g_{*}}\right)^{\frac{1}{2}}\left(\frac{M_{1}}{\mathrm{GeV}}\right)^{2} \frac{\mathrm{GeV}}{M_{P l}}\right]^{\frac{1}{n_{\mathcal{T}}}-1}
$$

According to recent results in literature, one has that $v \geqslant 1$, i.e., $n_{\mathcal{T}} \leqslant 2 / 3$, in order that the gravitino problem can be solved in non-standard cosmologies, and that the BBN temperature is $T_{B B N} \simeq(10-50)$ $\mathrm{GeV}$, so that the enhancement to the annihilation cross section is able to reproduce the observed Dark Matter density [63]. We discuss the case $n_{\mathcal{T}}=2 / 3$, i.e., $v=1$, and $n_{\mathcal{T}}<2 / 3$, i.e., $v>1$. In Table 1 are reported the values of $\left\{n_{\mathcal{T}}, \beta_{\mathcal{T}}, M_{1}\right\}$ such that the relation $T_{*}<M_{1} \lesssim T_{f}$, see Equation (11), is fulfilled. We have taken $M_{1}=\left(10^{8}, 10^{9}\right) \mathrm{GeV}$ (values $M_{1} \gtrsim 10^{10} \mathrm{GeV}$ are difficult to accommodate in this scenario). We see, for example, that the parameter $n_{\mathcal{T}}$ may vary in the range $0.65 \lesssim n_{\mathcal{T}} \lesssim 2 / 3$ as $M_{1}=10^{9} \mathrm{GeV}$, for a temperature $T_{*} \gtrsim \mathcal{O}(10 \mathrm{MeV})$. Notice that a large value of $A(T)$ also occurs in scalar tensor theories [24]. Although very tiny, there is a range of parameters $\left\{n_{\mathcal{T}}, \beta_{\mathcal{T}}\right\}$ for which the 
constraints from thermal leptogenesis (for massive degenerate neutrinos) are consistent with the ones needed to solve the gravitino problem (see Section 2.1).

Table 1. Values of the parameters $\left\{n_{\mathcal{T}}, \beta_{\mathcal{T}}, M_{1}\right\}$ such that $T_{*}<M_{1} \lesssim T_{f}$, according to Equation (11). Here we take $M_{1}=\left(10^{8}, 10^{9}\right) \mathrm{GeV}$ and $\tilde{\beta} \mathcal{T} \equiv \beta_{\mathcal{T}} / \mathrm{GeV}^{2\left(1-n_{\mathcal{T}}\right)}$.

\begin{tabular}{llll}
\hline$\left\{n_{\mathcal{T}}, \tilde{\beta}_{\mathcal{T}}, M_{\mathbf{1}}(\mathrm{GeV})\right\}$ & $T_{*}(\mathrm{GeV})$ & $T_{f}(\mathrm{GeV})$ & $A\left(T=M_{\mathbf{1}}\right)$ \\
\hline$\left\{2 / 3,10^{-16}, 10^{9}\right\}$ & 0.038 & $1.39 \times 10^{9}$ & $2.5 \times 10^{10}$ \\
$\left\{0.67,10^{-16}, 10^{9}\right\}$ & 0.029 & $1.87 \times 10^{9}$ & $2.3 \times 10^{10}$ \\
$\left\{0.66,10^{-17}, 10^{9}\right\}$ & 0.012 & $1.80 \times 10^{9}$ & $1.7 \times 10^{11}$ \\
$\left\{0.65,10^{-18}, 10^{9}\right\}$ & 0.005 & $1.68 \times 10^{9}$ & $1.4 \times 10^{12}$ \\
\hline$\left\{2 / 3,10^{-16}, 10^{8}\right\}$ & 0.038 & $1.39 \times 10^{9}$ & $2.5 \times 10^{9}$ \\
$\left\{0.62,10^{-18}, 10^{8}\right\}$ & 0.050 & $1.4 \times 10^{8}$ & $2.5 \times 10^{11}$ \\
$\left\{0.615,10^{-18}, 10^{8}\right\}$ & 0.070 & $1.01 \times 10^{8}$ & $2.8 \times 10^{11}$ \\
\hline
\end{tabular}

\subsection{Shear Dominated Universe}

The shear dominated Universe, or Bianchi's type I Universe, is a Universe homogeneous but anisotropic, characterized by three time dependent scale factors $a_{i}(t), i=1,2,3$, one for each spatial directions. For $a_{1}=a_{2}=a_{3}$ one recovers the standard FRW cosmology. The Friedman equation for this Universe reads [64-66].

$$
H^{2}=\frac{8 \pi}{3 M_{P l}^{2}}\left(\rho+\rho_{s}\right)
$$

where $\rho=\frac{\pi^{4} g_{*} T^{4}}{30}$, and $\rho_{s}$ is the shear energy density

$$
\rho_{s}=\frac{M_{P l^{2}}}{48 \pi}\left[\left(H_{1}-H_{2}\right)^{2}+\left(H_{1}-H_{3}\right)^{2}+\left(H_{2}-H_{3}\right)^{2}\right]
$$

where $H_{i}=\frac{\dot{a}_{i}}{a_{i}}, i=1,2,3$, are the expansion rate of the three scale factors. Expressing results in terms of the temperature $T$, one finds that the expansion rate of the Universe can be written in the form

$$
H(T)=H_{G R} \sqrt{1+\frac{g_{*}}{g_{*}^{e}} \frac{T^{2}}{T_{e}^{2}}},
$$

where $T_{e}$ is the temperature such that $\rho=\rho_{s}$ (it is related to the transition temperature $T_{*}$ as we will see in a moment), and $g_{*}^{e}$ is the number of degree of freedom evaluated at $T_{e}$. The Universe is therefore shear dominated when $T \gg T_{e}$ (before the BBN), while for $T \ll T_{e}$, the Universe is radiation dominated and the background evolves according to the standard cosmological model. BBN constraints require $T_{e} \gtrsim 2.5 \mathrm{MeV}$. During the shear dominated phase $T \gg T_{e}$ the factor $A(T)$ reads

$$
A(T)=\sqrt{1+\frac{g_{*}}{g_{*}^{e}}\left(\frac{T}{T_{e}}\right)^{2}} \simeq \frac{g_{*}}{g_{*}^{e}} \frac{T}{T_{e}},
$$

From (25) it follows $\eta_{*}=1, v=1$ and $T_{*}=\frac{g_{*}^{e}}{g_{*}} T_{e}$. Requiring that $A\left(T=M_{1}\right)>1$ and $g_{*}^{e}=g_{*}$, one gets $T_{*} \lesssim 10^{-2} M_{1}$, and $T_{f} \lesssim 2.5 \times 10^{7} \sqrt{M_{1} \mathrm{GeV}}$. Taking, for example, $A\left(M_{1}\right) \simeq 10^{2}$ and the lower bound on Majorana heavy neutrino $M_{1}=10^{9} \mathrm{GeV}$, it follows $T_{f} \simeq 0.8 \times 10^{12} \mathrm{GeV}$ and $T_{*} \simeq 10^{7} \mathrm{GeV}$ (according to (11)). The latter value for the transition temperature $T_{*} \sim 10^{7} \mathrm{GeV}$ allows in turn to solve the gravitino problem, as discussed in Section 2.1.

\section{Conclusions}

The cosmological evolution of the Universe is, at the moment, observationally tested at temperature $T \sim 1 \mathrm{MeV}$, and for $T>1 \mathrm{MeV}$ it is not excluded that new physics (non-standard 
cosmology, Dark Matter, Dark Energy, and so on) might be occurred opening a serious of questions which are still under a deep scrutiny.

In this paper we focused on some consequences of non-standard cosmologies in the framework of thermal Leptogenesis and gravitino late abundance. Since the out-of-equilibrium condition gets modified whether or not the cosmic evolution of the Universe is described by non-standard cosmology, then the consistence with thermal Leptogenesis, in the case of degenerate mass spectrum of light neutrinos, constrains the parameters space of these cosmological models. We have studied also the possibility that these constraints can solve the gravitino problem. As specific examples, we have considered the $f(\mathcal{T})$ cosmology and the shear dominated Universe.

Results derived in this paper show once more the intriguing interplay between particle physics and the non standard cosmological models needed for a consistent description of the evolution of the very early Universe.

Author Contributions: The authors conceived the idea, developed the theory, and performed the theoretical analyses. All authors have read and agreed to the published version of the manuscript.

Acknowledgments: A.C., G.L. and A.Q. acknowledges partial financial support from MIUR, INFN and COST Action CA1511 Cosmology and Astrophysics Network for Theoretical Advances and Training Actions (CANTATA). S.M.G. acknowledges support from the European Regional Development Fund the Competitiveness and Cohesion Operational Programme (KK.01.1.1.06-RBI TWIN SIN), the Croatian Science Fund Project No. IP-2016-6-3347 and IP-2019-4-3321 and the QuantiXLie Center of Excellence (Grant KK.01.1.1.01.0004).

Conflicts of Interest: The authors declare no conflicts of interest.

\section{Appendix A. Thermal Leptogenesis Scenario}

In this Appendix, we shortly recall the main features of thermal Leptogenesis. The Lagrangian density is $[67,68]$

$$
\mathcal{L}=h_{\beta}^{*}\left(\bar{L}_{\beta} \phi^{c *}\right) E_{\beta}-\lambda_{\alpha k}^{*}\left(\bar{L}_{\alpha} \phi^{*}\right) N_{k}-\frac{1}{2} \bar{N}_{j} M_{j} N_{j}^{c}+\text { h.c. },
$$

where $L=(e v)_{L}^{T}$ is the Standard Model left-handed doublet, $E=e_{R}$ is the right-handed singlet, $\phi$ is the scalar field, $N_{j}$ are the singlet fermions (Majorana neutrinos), $\alpha, \beta$ are the flavor indices of the Standard Model $(\alpha, \beta=e, \mu, \tau), M$ is the mass matrix, $h$ are coupling constants, and finally $\lambda$ are Yakawa matrices. Equation (A1) is written in a basis such that $h$ and $M$ are real and diagonal, and $\lambda$ is complex. Integrating out the heavy fermions $N_{i}$, one infers the effective light neutrino masses (see-saw mechanism): $m_{v \alpha \beta}=\lambda_{\alpha k} M_{k}^{-1} \lambda_{\beta k}$.

Consider, for simplicity, the lightest Majorana singlet $N_{1}$ (with $M_{1} \ll M_{2,3}$ ). The allowed channel decays are $N_{1} \rightarrow L_{\alpha}+\phi$ and $N_{1} \rightarrow \bar{L}_{\alpha}+\phi^{\dagger}$. The net lepton asymmetry is given by $[67,68]$

$$
\eta \simeq \frac{\epsilon}{g_{*}} \eta_{e f f}
$$

Here $\eta_{e f f}$ is the efficiency factor. It may assume values in the range $0<\eta_{e f f}<1$. In fact, define the freeze-out temperature $T_{F}$ as the temperature for which $\left.\Gamma\left(\phi+L \rightarrow N_{1}\right)\right|_{T_{F}}<H\left(T_{F}\right)$, where $\Gamma\left(\phi+L \rightarrow N_{1}\right) \simeq \frac{1}{2} \Gamma_{N_{1}} e^{-M_{1} / T}$, with $\Gamma_{N_{1}}=\frac{\left(\lambda^{\dagger} \lambda\right)_{11} M_{1}}{8 \pi}$ and, in the standard cosmological model, $H=\left(\frac{8 \pi}{3 M_{P}^{2}} \rho\right)^{1 / 2}=1.66 g_{*}^{1 / 2} \frac{T^{2}}{M_{P}}$. Below $T_{F}$ the density of the fermion $N_{1}$ is Boltzmann suppressed $\left(N_{1} \sim e^{-M_{1} / T}\right)$, so that the decay of $N_{1}$ at $T<T_{F}$ contributes to the lepton asymmetry, with 
$\eta_{\text {eff }} \simeq \frac{n_{N_{1}}\left(T_{F}\right)}{n_{N_{1}}\left(T \gg M_{1}\right)} \simeq e^{-M_{1} / T_{F}}<1$. The efficiency factor depends on how much the $N_{1}$ decays are out-of-equilibrium and $\epsilon$ is the $C P$ parameter related to the asymmetry in the $N_{1}$ decays

$$
\begin{aligned}
\epsilon & \equiv \frac{\Gamma\left(N_{1} \rightarrow \phi+L\right)-\Gamma\left(N_{1} \rightarrow \phi^{\dagger}+\bar{L}\right)}{\Gamma\left(N_{1} \rightarrow \phi+L\right)+\Gamma\left(N_{1} \rightarrow \phi^{\dagger}+\bar{L}\right)} \\
& \simeq 10^{-6} \sin \delta \frac{m_{3}}{5 \times 10^{-2} \mathrm{eV}} \frac{M_{1}}{10^{10} \mathrm{GeV}},
\end{aligned}
$$

where $\delta$ is the phase coming from the complex Yukawa couplings, $m_{3}$ the heaviest light neutrino mass normalized to the atmospheric neutrino oscillation data $\sqrt{\Delta m_{\oplus}} \simeq 0.05 \mathrm{eV}$ [69], and $M_{1} \ll M_{j}, j=2,3$. This quantity is generated from the interference of the tree-level and one-loop amplitudes, with complex Yakawa couplings. From Equation (A2) it follows that the observed baryon asymmetry $\left(\eta \sim 10^{-10}\right)$ provides a lower bound on the mass of the Majorana neutrino $N_{1}$ given by $M_{1} \gtrsim\left(10^{8}-10^{9}\right) \mathrm{GeV}$ [47]. In terms of the scaled quantities (3) and (4), the condition of out-of-equilibrium decay $(\Gamma(T)<H(T)$ ) can be cast in the form $\tilde{m}_{1}<m_{*}$. This equation provides an upper bound on the lightest neutrino mass $m^{v}$ being $m^{v} \lesssim \tilde{m}_{1}[46,47]$.

\section{References}

1. Starobinsky, A.A. A New Type of Isotropic Cosmological Models without Singularity. Phys. Lett. B 1980, 91, 99. [CrossRef]

2. Nojiri, S.; Odintsov, S.D.; Oikonomou, V.K. Modified Gravity Theories on a Nutshell: Inflation, Bounce and Late-time Evolution. Phys. Rep. 2017, 692, 1-104. [CrossRef]

3. Sami, M. Models of dark energy. Lect. Notes Phys. 2007, 720, 219.

4. Sawicki, I.; Hu, W. Stability of Cosmological Solution in $\mathrm{f}(\mathrm{R})$ Models of Gravity. Phys. Rev. D 2007, 75, 127502. [CrossRef]

5. Li, B.; Barrow, J.D.; Mota, D.F. The Cosmology of Modified Gauss-Bonnet Gravity. Phys. Rev. D 2007, 76, 044027. [CrossRef]

6. Barrow, J.D. Scalar-tensor cosmologies. Phys. Rev. D 1993, 47, 5329. [CrossRef]

7. Clifton, T.; Barrow, J.D. The Power of General Relativity. Phys. Rev. D 2005, 72, 103005. [CrossRef]

8. Capozziello, S.; Lambiase, G. Higher order corrections to the effective gravitational action from Noether symmetry approach. Gen. Relativ. Gravit. 2000, 32, 295-311. [CrossRef]

9. Capozziello, S.; Lambiase, G. Nonminimal derivative coupling and the recovering of cosmological constant. Gen. Relativ. Gravit. 1999, 31, 1005. [CrossRef]

10. Capozziello, S.; Lambiase, G.; Schmidt, H.J. Nonminimal derivative couplings and inflation in generalized theories of gravity. Ann. Phys. 2000, 9, 39-48. [CrossRef]

11. Nojiri, S.; Odintsov, S.D. Inhomogeneous equation of state of the universe: Phantom era, future singularity and crossing the phantom barrier. Phys. Rev. D 2005, 72, 023003. [CrossRef]

12. Chakravarty, G.K.; Mohanty, S.; Lambiase, G. Testing theories of gravity and supergravity with inflation and observations of the cosmic microwave background. Int. J. Mod. Phys. D 2017, 26, 1730023. [CrossRef]

13. Capolupo, A.; Capozziello, S.; Vitiello, G. Neutrino mixing as a source of dark energy. Phys. Lett. A 2007, 363, 53-56. [CrossRef]

14. Capolupo, A.; Capozziello, S.; Vitiello, G. Dark energy and particle mixing. Phys. Lett. A 2007, 373, 601-610. [CrossRef]

15. Capolupo, A. Dark matter and dark energy induced by condensates. Adv. High Energy Phys. 2016, 2016, 8089142. [CrossRef]

16. Capolupo, A. Cosmological Effects of Quantum Vacuum Condensates. Galaxies 2017, 5, 98. [CrossRef]

17. Capolupo, A. Quantum vacuum, dark matter, dark energy and spontaneous supersymmetry breaking. Adv. High Energy Phys. 2018, 2018, 9840351. [CrossRef]

18. Capolupo, A.; De Martino, I.; Lambiase, G.; Stabile, A. Axion-photon mixing in quantum field theory and vacuum energy. Phys. Lett. B 2019, 790, 427. [CrossRef]

19. Birrell, N.D.; Davies, P.C.W. Quantum Fields in Curved Space; Cambridge University Press: Cambridge, UK, 1982. 
20. Buchbinder, I.L.; Odintsov, S.D.; Shapiro, I.L. Effective Action in Quantum Gravity; IOP: Bristol, UK, 1992.

21. Barth, N.H.; Christensen, S. Quantizing Fourth Order Gravity Theories. 1. The Functional Integral. Phys. Rev. D 1983, 28, 8. [CrossRef]

22. Riess, A.G.; Filippenko, A.V.; Challis, P.; Clocchiatti, A.; Diercks, A.; Garnavich, P.M.; Gilliland, R.L.; Hogan, C.J.; Jha, S.; Kirshner, R.P.; et al. Observational Evidence from Supernovae for an Accelerating Universe and a Cosmological Constant. Astron. J. 1998, 116, 1009. [CrossRef]

23. Perlmutter, S.; Aldering, G.; Della Valle, M.; Deustua, S.; Ellis, R.S.; Fabbro, S.; Fruchter, A.; Goldhaber, G.; Groom, D.E.; Hook, I.M.; et al. Discovery of a Supernova Explosion at Half the Age of the Universe and its Cosmological Implications. Nature 1998, 391, 51. [CrossRef]

24. Schelke, M.; Catena, R.; Fornengo, N.; Masiero, A.; Pietroni, M. Constraining pre Big-Bang-Nucleosynthesis Expansion using Cosmic Antiprotons. Phys. Rev. D 2006, 74, 083505. [CrossRef]

25. Gelmini, G.; Gondolo, P. DM Production Mechanisms; Bertone, G., Ed.; Particle dark matter 121-141; Cambridge University Press: Cambridge, UK, 2010; arXiv:1009.3690.

26. Randal, L.; Sundrum, R. An Alternative to Compactification. Phys. Rev. Lett. 1991, 83, 4690. [CrossRef]

27. Profumo, S.; Ullio, P. SUSY dark matter and quintessence. J. Cosmol. Astropart. Phys. 2003, $0311,006$. [CrossRef]

28. Catena, R.; Fornengo, N.; Masiero, A.; Pietroni, M.; Schelke, M. Enlarging mSUGRA parameter space by decreasing pre-BBN Hubble rate in Scalar-Tensor Cosmologies. JHEP J. High Energy Phys. 2008, 10, 003. [CrossRef]

29. Catena, R.; Fornengo, N.; Pato, M.; Pieri, L.; Masiero, A. Thermal Relics in Modified Cosmologies: Bounds on Evolution Histories of the Early Universe and Cosmological Boosts for PAMELA. Phys. Rev. D 2010, 81, 123522. [CrossRef]

30. Kamionkowski, M.; Turner, M.S. Thermal Relics: Do We Know Their Abundances? Phys. Rev. D 1990, 42, 3310. [CrossRef]

31. Santiago, D.I.; Kalligas, D.; Wagoner, R.V. Scalar-tensor cosmologies and their late time evolution. Phys. Rev. D 1998, 58, 124005 . [CrossRef]

32. Salati, P. Quintessence and the relic density of neutralinos. Phys. Lett. B 2003, 571, 121. [CrossRef]

33. Lambiase, G.; Mohanty, S.; Stabile, A. PeV IceCube signals and Dark Matter relic abundance in modified cosmologies. Eur. Phys. J. C 2018, 78, 350. [CrossRef]

34. Buoninfante, L.; Lambiase, G. Cosmology with bulk viscosity and the gravitino problem. Eur. Phys. J. C 2017, 77, 287. [CrossRef]

35. Chung, D.J.H.; Freese, K. Cosmological challenges in theories with extra dimensions and remarks on the horizon problem. Phys. Rev. D 2000, 61, 023511. [CrossRef]

36. Catena, R.; Fornengo, N.; Masiero, A.; Pietroni, M.; Rosati, F. Dark matter relic abundance and scalar-tensor dark energy. Phys. Rev. D 2004, 70, 063519. [CrossRef]

37. Ferraro, R.; Fiorini, F. Modified teleparallel gravity: Inflation without inflaton. Phys. Rev. D 2007, 75, 084031. [CrossRef]

38. Lambiase, G. Dark matter relic abundance and big bang nucleosynthesis in Horava's gravity. Phys. Rev. D 2011, 83, 107501. [CrossRef]

39. Capozziello, S.; Lambiase, G.; Saridakis, E.N. Constraining $f(T)$ teleparallel gravity by Big Bang Nucleosynthesis. Eur. Phys. J. C 2017, 77, 576. [CrossRef]

40. Fukugita, M.; Yanagida, T. Baryogenesis Without Grand Unification. Phys. Lett. B 1986, 174, 45-47. [CrossRef]

41. Kuzmin, V.A.; Rubakov, V.A.; Shaposhnikov, M.E. On the Anomalous Electroweak Baryon Number Nonconservation in the Early Universe. Phys. Lett. B 1985, 155, 36. [CrossRef]

42. Fong, C.S.; Nardi, E.; Riotto, A. Leptogenesis in the Universe. Adv. High Energy Phys. 2012, $2012,158303$. [CrossRef]

43. Buchmuller, W.; Di Bari, P.; Plumacher, M. Leptogenesis for pedestrians. Ann. Phys. 2005, 315, 305. [CrossRef]

44. Lambiase, G.; Mohanty, S.; Prasanna, A.R. Neutrino coupling to cosmological background: A review on gravitational Baryo/Leptogenesis. Int. J. Mod. Phys. D 2013, 22, 1330030. [CrossRef]

45. Okada, N.; Seto, O. Thermal leptogenesis in brane world cosmology. Phys. Rev. D 2006, 73, 063505. [CrossRef]

46. Fuji, M.; Hamaguchi, K.; Yanagida, T. Leptogenesis with almost degenerate majorana neutrinos. Phys. Rev. D 2002, 65, 115012. [CrossRef] 
47. Davidson, S.; Ibarra, A. A Lower bound on the right-handed neutrino mass from leptogenesis. Phys. Lett. $B$ 2002, 535, 25-32. [CrossRef]

48. Fischler, W.; Giudice, G.F.; Leigh, R.G.; Paban, S. Constraints on the baryogenesis scale from neutrino masses. Phys. Lett. B 1991, 258, 45-48. [CrossRef]

49. Buchmuller, W.; Yanagida, T. Baryogenesis and the scale of B-L breaking. Phys. Lett. B 1993, 302, $240-244$. [CrossRef]

50. Khlopov, M.Y.; Linde, A.D. Is It Easy to Save the Gravitino? Phys. Lett. B 1984, 138, 265-268. [CrossRef]

51. Yu Khlopov, M.; Barrau, A.; Grain, J. Gravitino production by primordial black hole evaporation and constraints on the inhomogeneity of the early universe. Class. Quant. Grav. 2006, 23, 1875. [CrossRef]

52. Buchmüller, W.; Di Bari, P.; Plumacher, M. Cosmic microwave background, matter-antimatter asymmetry and neutrino masses. Nucl. Phys. B 2002, 643, 367. [CrossRef]

53. Ellis, J.R.; Kim, J.E.; Nanopoulos, D.V. Cosmological Gravitino Regeneration and Decay. Phys. Lett. B 1984, 145, 181. [CrossRef]

54. Cyburt, R.H.; Ellis, J.R.; Fields, B.D.; Olive, K.A. Updated nucleosynthesis constraints on unstable relic particles. Phys. Rev. D 2003, 67, 103521. [CrossRef]

55. Kawasaki, M.; Kohri, K.; Moroi, T. Adronic decay of late-decaying particles and Big-Bang Nucleosynthesis. Phys. Lett. B 2005, 625, 7. [CrossRef]

56. Iminniyaz, H.; Salai, B.; Lv, G.-L. Relic Density of Asymmetric Dark Matter in Modified Cosmological Scenarios. Commun. Theor. Phys. 2018, 70, 602. [CrossRef]

57. Poulin, A. Dark Matter freezeout in modified cosmological scenarios. Phys. Rev. D 2019, 100, 043022. [CrossRef]

58. Linder, E.V. Einstein's other gravity and the acceleration of the Universe. Phys. Rev. D 2010, 81, 127301. [CrossRef]

59. Aldrovandi, R.; Pereira, J.G. Teleparallel Gravity: An Introduction; Springer: Dordrecht, The Netherlands, 2013.

60. Cai, Y.F.; Capozziello, S.; De Laurentis, M.; Saridakis, E.N. $f(T)$ teleparallel gravity and cosmology. Rep. Prog. Phys. 2016, 79, 106901. [CrossRef] [PubMed]

61. Nesseris, S.; Basilakos, S.; Saridakis, E.N.; Perivolaropoulos, L. Viable $f(T)$ models are practically indistinguishable from $\Lambda$ CDM. Phys. Rev. D 2013, 88, 103010. [CrossRef]

62. Ferraro, R.; Fiorini, F. On Born-Infeld Gravity in Weitzenbock spacetime. Phys. Rev. D 2008, 78, 124019. [CrossRef]

63. D'Eramo, F.; Fernandez, N.; Profumo, S. When the Universe Expands Too Fast: Relentless Dark Matter. J. Cosmol. Astropart. Phys. 2017, 2017, 012. [CrossRef]

64. Ellis, G.F.R.; MacCallum, M.A.H. A Class of homogeneous cosmological models. Commun. Math. Phys. 1969, 12, 108-141. [CrossRef]

65. Ryan, L.C. Shepley, Homogenous Relativistic Cosmologies; Princeton University Press: Princeton, NJ, USA, 1975.

66. Ellis, G.F.R.; van Elst, H. Cosmological models (Cargèse lectures 1998). NATO Adv. Study Inst. Ser. C. Math. Phys. Sci. 1999, 541, 1-116.

67. Nir, Y. Introduction to leptogenesis. arXiv 2007, arXiv:hep-ph/0702199.

68. Covi, L.; Roulet, E.; Vissani, F. CP violating decays in leptogenesis scenarios. Phys. Lett. B 1996, $384,169$. [CrossRef]

69. Ashie, Y.; Hosaka, J.; Ishihara, K.; Itow, Y.; Kameda, J.; Koshio, Y.; Minamino, A.; Mitsuda, C.; Miura, M.; Moriyama, S.; et al. Measurement of atmospheric neutrino oscillation parameters by Super-Kamiokande I. Phys. Rev. D 2005, 71, 112005. [CrossRef]

(C) 2020 by the authors. Licensee MDPI, Basel, Switzerland. This article is an open access article distributed under the terms and conditions of the Creative Commons Attribution (CC BY) license (http://creativecommons.org/licenses/by/4.0/). 